\title{
Comparative Analysis of Tongue Indices between Patients with and without a Self-Reported Yin Deficiency: A Cross-Sectional Study
}

\author{
Su-Ryun Kim, ${ }^{1}$ Woojin Choi, ${ }^{2}$ Inkwon Yeo, ${ }^{3}$ and Dong-Hyun Nam $^{1}$ \\ ${ }^{1}$ Department of Biofunctional Medicine and Diagnosis, College of Korean Medicine, Sangji University, Wonju 26382, \\ Republic of Korea \\ ${ }^{2}$ Department of Neuropsychiatry, College of Korean Medicine, Sangji University, Wonju 26382, Republic of Korea \\ ${ }^{3}$ Department of Statistics, Sookmyung Women's University, Seoul 04310, Republic of Korea
}

Correspondence should be addressed to Dong-Hyun Nam; omdnam@naver.com

Received 2 January 2017; Accepted 19 April 2017; Published 17 May 2017

Academic Editor: Seong S. Nah

Copyright (C) 2017 Su-Ryun Kim et al. This is an open access article distributed under the Creative Commons Attribution License, which permits unrestricted use, distribution, and reproduction in any medium, provided the original work is properly cited.

\begin{abstract}
We investigated the hypothesis that Yin-deficient patients have a reddened tongue with less coating. We screened 189 participants aged 20 to 49 years, complaining of headache. To classify patients in terms of Yin deficiency, we used two self-reporting Yindeficiency questionnaires (Yin-Deficiency Questionnaire and Yin-Deficiency Scale) and diagnosis by a doctor. Based on the tests, a total of 33 subjects were assigned to a Yin-deficient group and 33 subjects were assigned to a nondeficient control group. Tongue images were acquired using a computerized tongue diagnostic system, for evaluating tongue indices. The tongue coating percentage and tongue redness were calculated as the mean $a^{*}$ value of both the whole tongue area (WT $a^{*}$ ) and the tongue body area (TB $\left.a^{*}\right)$. The tongue coating percentage of the Yin-deficient group $(34.79 \pm 10.76)$ was lower than that of the nondeficient group (44.13 $\pm 14.08)$. The WT $a^{*}$ value of the Yin-deficient group $(19.39 \pm 1.52)$ was significantly higher than that of the nondeficient group $(18.21 \pm 2.06)$. However, the difference in the TB $a^{*}$ value between the two groups was not significant. In conclusion, we verified that Yin-deficient patients had less tongue coating and tended to have a more reddish tongue than nondeficient patients.
\end{abstract}

\section{Introduction}

In traditional Korean medicine (TKM) and traditional Chinese medicine (TCM), pattern identification is used to determine the pathological state of a patient. The patterns, called Zheng, imply the cause, nature, location, and the severity of the disease. Thus, a traditional medicine practitioner gathers and analyzes the symptoms and signs present in a patient to determine the course of action for treating an underlying disease. To identify such patterns, practitioners typically perform inspections that involve listening, smelling, inquiry, and palpation [1].

Inspection of the tongue is an important diagnostic approach in pattern identification in TKM because the tongue is considered to reflect the status of the human body, such as that of the qi-blood and the fluid of the internal organs, rapidly [2]. The practitioner examines the color, shape, moisture, and movement of the tongue body (which is a composite of muscles and vessels) and the color, thickness, and distribution of the tongue coating (a fur-like attachment to the dorsum of the tongue).

A Yin-deficiency (YD) pattern is a pathological state. The clinical symptoms of YD are typically associated with emaciation, tidal fever, night sweats, malar flush, palpitations, insomnia, dizziness, tinnitus, dry mouth and throat, constipation, a reddened tongue with little coating, and a fine rapid pulse [3]. In particular, a reddened tongue with little coating is regarded as a distinctive sign of YD. A "reddened tongue" sign indicates that the color of the tongue is reddish rather than the normal pale red color of the tongue, and a tongue with "little coating" indicates that either no or only a small amount of tongue coating is visible on the tongue body.

However, whether the sign of a reddened tongue with little coating is associated with YD in patients remains 
unverified. Moreover, the definition of a "reddened tongue" has not been definitively established. A "reddened tongue" can be interpreted as the result of less coating on the tongue body, which reveals a pinker or redder color, or may reflect increased blood flow.

Several TKM and TCM studies [4-7] have investigated the tongue status of Yin-deficient patients. In those studies, the Yin-deficient patients were mainly grouped at the discretion of the practitioners; this methodology lacked objectivity. Thus, the results of these studies are not highly reliable. For objective assessment of a Yin-deficient status, several tools have been developed, such as the Yin-Deficiency Questionnaire (YDQ), the Yin-Deficiency Scale (YDS), Shi-pattern analysis, the Yin/Yang-deficiency Questionnaire, and the YinXu Body Constitution Questionnaire [8]. Nevertheless, most of these tools do not include a tongue inspection or rely only on the subjective tongue inspection of the practitioner [911]. Some studies [12-15] have reported that inspection of the tongue has a low reliability, and thus an objective approach to inspection is needed. Therefore, several types of quantitative tongue assessment systems, such as a computerized tongue diagnostic system (CTDS), have been developed to solve this problem. Han et al. [16] investigated the relationship between tongue status and YD with the YDQ, using a CTDS; the results of this study were not consistent with the traditional medicine theory of tongue diagnosis. However, a single investigation is not sufficient to gain an understanding of the relationship between tongue status and YD.

Therefore, the present study quantitatively investigated the hypothesis that YD in patients is associated with a reddened tongue with little coating. We compared evaluation indices of the tongue between a Yin-deficient group of patients and a nondeficient control group. To identify patients with a YD and nondeficient patients, we used two selfreporting questionnaires, the YDS and YDQ [1, 17], both of which have been previously validated. To investigate differences in the tongue status between the two patient groups, we evaluated indices of the amount of tongue coating and the tongue color using a CTDS.

\section{Materials and Methods}

2.1. Ethics Approval and Consent to Participate. This study was conducted according to the standards of the International Committee on Harmonization of Good Clinical Practice and the revised version of the Declaration of Helsinki. Written informed consent was obtained from all of the study participants before the experiment. The Institutional Review Board of the Traditional Korean Medicine Hospital of Sangji University in Wonju, Republic of Korea, approved the experimental protocol (IRB number SJ IRB-Human-15-010).

2.2. Study Design. Between December 2015 and August 2016, a total of 189 people were screened from among the outpatients complaining of headache at the Traditional Korean Medicine Hospital of Sangji University in Wonju, Republic of Korea. All patients were aged between 20 and 49 years. The exclusion criteria were pregnancy, severe systemic organ diseases, use of drugs within the 7 days prior to screening, smoking, vitamin B use, or an abnormal condition of the tongue, that is, the inability to open the jaw and protrude the tongue stably, the presence of a geographic tongue, bleeding, malformation of the tongue, or the presence of dental braces. Thirty-one patients were excluded in the process of screening according to these criteria.

Demographic characteristics were recorded for each patient. In addition, the duration of the headache was recorded and severity of the headache was also assessed using a visual analog scale. After an experimenter explained the YDS and YDQ, each patient completed the questionnaires. To prevent any bias due to a misunderstanding of the questionnaires, a TKM doctor with more than 10 years of clinical experience diagnosed each patient as Yin-deficient or nondeficient, while blinded to the results of the questionnaires and without inspection of the patient's tongue. The diagnosis of a YD or a nondeficient status in patients was thus based on the scores of the YDS and the YDQ as well as the independent opinion of the doctor. Fiftynine patients were excluded because of discordance between the two questionnaires. One YD subject was also excluded from our study based on the decision of the doctor that he was nondeficient. Then, the nondeficient patient group was matched to the group of patients who had YD, in terms of age and gender. Thirty-two nondeficient individuals were also excluded for matching. Finally, 66 participants were registered for this study. The overall design of the study is presented in Figure 1.

2.3. Tongue Examination. All subjects had their tongues examined using a CTDS. The subjects were required to avoid food and liquid intake for at least $4 \mathrm{~h}$ before the tongue examination and to refrain from brushing their teeth and tongue. In consideration of the influence of circadian rhythms, the tongue examination was conducted in the morning within $24 \mathrm{~h}$ of the screening. To image the tongue, the patients were instructed on how to touch their faces and protrude their tongues to the CTDS. After the patient exercised the procedure several times, opening their mouths and extruding the tongue to reveal their entire tongue, the CTDS was used to acquire an image of the tongue. To assess the reliability of the CTDS, an image of the tongue was acquired again in the same manner after $30 \mathrm{~min}$. After image acquisition, we assessed whether the patients experienced any adverse event. The initial 66 images that were acquired during this examination were analyzed in a comparison of the YD patient group and the nondeficient patient group. The second group of images was only used to calculate the reliability of the CTDS.

2.4. The Yin-Deficiency Questionnaire and the Yin-Deficiency Scale. In 2007, the YDQ was developed for objective measurement of the severity of a YD [1]. The reliability and validity of the YDQ have been previously established [18], and the YDQ has been used to investigate the relationship between a YD and other symptoms, such as dry mouth, hot flush, and skin disorders [19-21]. The YDQ is comprised of 10 questions to which the patients responded by marking the 


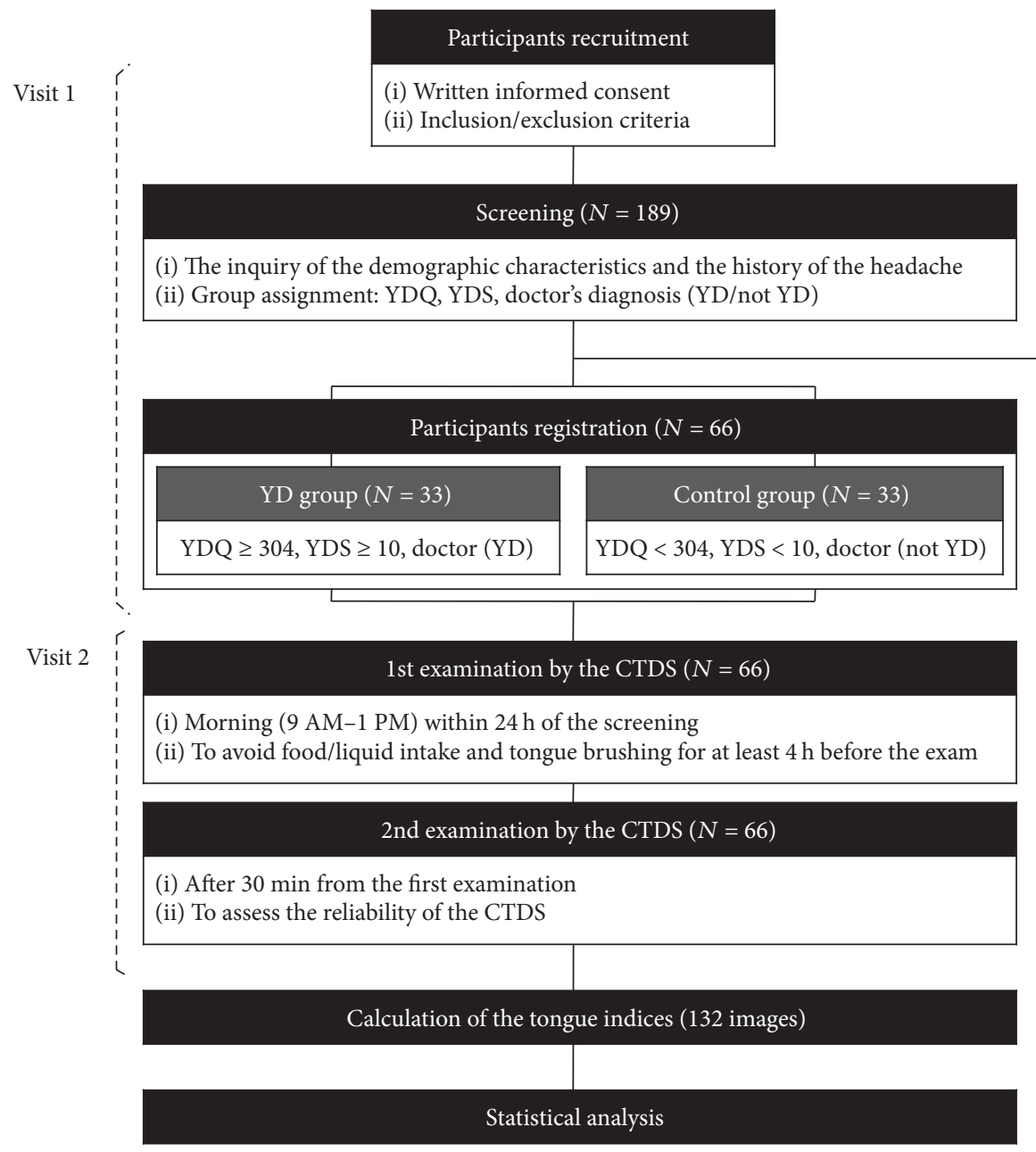

FIGURE 1: Flow chart of the experimental design of the present study. YDS: Yin-Deficiency Scale; YDQ: Yin-Deficiency Questionnaire; YD: Yin deficiency; CTDS: computerized tongue diagnostic system.

severity of their symptoms on a $100 \mathrm{~mm}$ bar. The cut-off score for a YD diagnosis is 304 [22].

Park et al. [17] developed the YDS, which suggests the optimum cut-off score for a diagnosis of YD as 10. The YDS includes a total of 27 questions to which the patients responded on a scale of 1 to 7 , to indicate the severity of a YD. To discriminate patients with YD, the score of the YDS was calculated on a dichotomous scale; the scores of 1, 2, 3, and 4 were converted to 0 points and the scores of 5,6 , and 7 were converted to 1 point.

2.5. Image Acquisition System. A CTDS (CTS-1000, Daiseung Medics, Seoul, South Korea) was used to acquire tongue images. The CTDS comprised a camera, illuminators, an external light shielding system, and analysis software. The camera was set up using a color board (Color Checker Passport, X-Rite, Grand Rapids, MI, USA) before the capture of tongue images. The operator captured an image of the tongue together with two $18 \%$ gray chips (R27, Kodak, Rochester, NY, USA) on the bilateral sides of the tongue, to evaluate the

\section{Excluded $(N=123)$}

(i) Exclusion criteria $(N=31)$

Medication $(N=1)$

Smoking $(N=13)$

Vitamin B $(N=6)$

Dental braces $(N=11)$

(ii) $\mathrm{YDQ} \neq \mathrm{YDS}(N=59)$

(iii) $\mathrm{YDQ}=\mathrm{YDS} \neq$ doctor's decision $(N=1)$

(iv) Age and gender mismatching $(N=32)$

\[ \text { Excluded }(N=123) \]
(i) Exclusion criteria $(N=31)$
Medication $(N=1)$
Smoking $(N=13)$
Vitamin $B(N=6)$
Dental braces $(N=11)$
(ii) YDQ $\neq$ YDS $(N=59)$
(iii) YDQ $=$ YDS $\neq$ doctor's decision $(N=1)$
(iv) Age and gender mismatching $(N=32)$

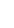




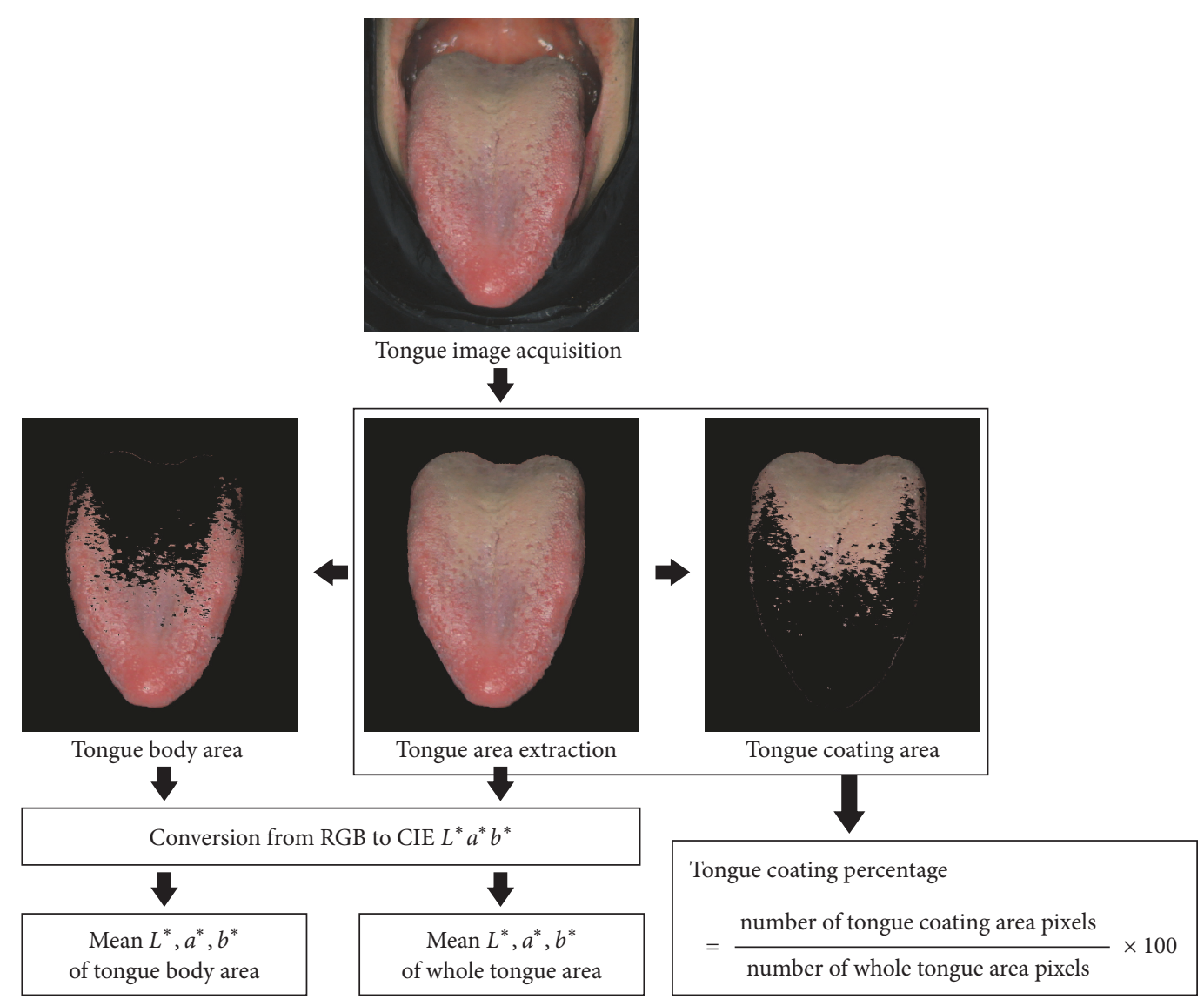

Figure 2: Calculation of tongue indices. The tongue area was extracted from an acquired tongue image. The tongue coating area was distinguished from the tongue body area based on the difference in color. The RGB color values in the areas of interest were converted into CIE- $L^{*} a^{*} b^{*}$ color values. Then, the mean $L^{*} a^{*} b^{*}$ values of whole tongue area and tongue body area were calculated. The tongue coating percentage was calculated as the percentage of the pixel number of the tongue coating area to the pixel numbers of the whole tongue area.

2.7. Statistical Analysis. The baseline characteristics of the participants are presented using descriptive statistics. Differences in the demographic characteristics and the severity of the headache between the YD group and nondeficient control group were compared using a chi-squared test for categorical data and an independent samples $t$-test for continuous data. If the assumption of normality was not confirmed, Mann-Whitney $U$ test was used. To evaluate the homogeneity of the headache duration, we used the proportional odds model [24]. Levene's test was performed to assess the equality of variances for the demographic characteristics and the tongue indices between the two groups. Intraclass correlation coefficients (ICCs) were calculated to assess the reliability of the CTDS. ICC values above 0.8 indicate acceptable reliability according to Shrout and Fleiss [25]. A binary logistic regression analysis was performed to predict the probability of YD occurrence according to the indices. Statistical analyses were performed using IBM SPSS Statistics 23 (IBM Corporation, Armonk, NY, USA) or SAS version 9.4 (SAS Institute Inc., Cary, NC, USA). A $p$ value $<0.05$ was considered statistically significant.

\section{Results}

3.1. Baseline Characteristics of the Participants. The baseline characteristics of the participants are shown in Table 1. The demographic differences between the YD group and nondeficient control group were not significant. In addition, differences in headache duration between the two groups were not significant; the estimated coefficient of the group effect was 0.484 (standard error $=0.45$ ) and the corresponding $p$ value was 0.280 . However, the headache severity of the YD group was significantly higher than that of the nondeficient group.

3.2. Tongue Indices. The results for all of the tongue indices between the YD group and nondeficient control group are presented in Table 2 . The tongue coating percentage of the YD group $(34.79 \pm 10.76)$ was significantly lower than that of the nondeficient group ( $44.13 \pm 14.08 ; p=0.004)$. The WT $a^{*}$ value was significantly different between the two groups ( $p=0.010)$; the WT $a^{*}$ value of the YD group $(19.39 \pm 1.52)$ was higher than that of the nondeficient group $(18.21 \pm 2.06)$. However, there was no significant difference in the TB $a^{*}$ 
TABLE 1: Baseline characteristics of the participants.

\begin{tabular}{|c|c|c|c|c|}
\hline & $\begin{array}{c}\text { Yin } \\
\text { deficiency } \\
(N=33)\end{array}$ & $\begin{array}{l}\text { Control } \\
(N=33)\end{array}$ & $\begin{array}{c}\text { Total } \\
(N=66)\end{array}$ & $p$ value \\
\hline $\operatorname{Sex}(M / F)^{(a)}$ & $\begin{array}{c}10(30.3) / 23 \\
(69.7)\end{array}$ & $\begin{array}{c}12(36.4) / 21 \\
(63.6)\end{array}$ & $\begin{array}{c}22(33.3) / 44 \\
(66.7)\end{array}$ & 0.602 \\
\hline Age (years) $)^{(\mathrm{b})}$ & $30.82 \pm 7.49$ & $30.82 \pm 8.71$ & $30.82 \pm 8.06$ & 1.000 \\
\hline Height $(\mathrm{cm})^{(\mathrm{c})}$ & $165.99 \pm 6.31$ & $165.97 \pm 9.27$ & $165.98 \pm 7.87$ & 0.993 \\
\hline Weight $(\mathrm{kg})^{(\mathrm{b})}$ & $63.67 \pm 12.85$ & $63.26 \pm 12.52$ & $63.46 \pm 12.59$ & 0.896 \\
\hline $\begin{array}{l}\text { Systolic blood pressure } \\
(\mathrm{mmHg})^{(\mathrm{b})}\end{array}$ & $107.61 \pm 8.51$ & $111.42 \pm 13.90$ & $109.52 \pm 11.59$ & 0.183 \\
\hline $\begin{array}{l}\text { Diastolic blood pressure } \\
(\mathrm{mmHg})^{(\mathrm{b})}\end{array}$ & $72.36 \pm 6.67$ & $74.30 \pm 10.82$ & $73.33 \pm 8.97$ & 0.385 \\
\hline Pulse rate $(\mathrm{bpm})^{(\mathrm{b})}$ & $68.36 \pm 10.98$ & $66.58 \pm 8.75$ & $67.47 \pm 9.89$ & 0.467 \\
\hline Temperature $\left({ }^{\circ} \mathrm{C}\right)^{(\mathrm{b})}$ & $36.46 \pm 0.19$ & $36.40 \pm 0.18$ & $36.43 \pm 0.18$ & 0.140 \\
\hline \multicolumn{5}{|l|}{ Headache duration } \\
\hline Duration < 1 week & $6(18.2)$ & $12(36.4)$ & \multirow{4}{*}{$0.48 \pm 0.45$} & \multirow{4}{*}{0.280} \\
\hline 1 week $\leq$ duration $<1$ month & $10(30.3)$ & $5(15.2)$ & & \\
\hline $\begin{array}{l}1 \text { month } \leq \text { duration }<6 \\
\text { months }\end{array}$ & $4(12.1)$ & $6(18.2)$ & & \\
\hline 6 months $\leq$ duration & $13(39.4)$ & $10(30.3)$ & & \\
\hline Headache VAS ${ }^{(\mathrm{b})}$ & $4.55 \pm 1.86$ & $3.42 \pm 1.95$ & $3.98 \pm 1.97$ & $0.020^{*}$ \\
\hline
\end{tabular}

TABLE 2: Tongue indices.

\begin{tabular}{|c|c|c|c|c|c|}
\hline \multirow{2}{*}{ Variables } & \multicolumn{2}{|c|}{ Levene's test } & \multirow{2}{*}{$\begin{array}{l}\text { Yin deficiency } \\
\quad(N=33)\end{array}$} & \multirow{2}{*}{$\begin{array}{l}\text { Control } \\
(N=33)\end{array}$} & \multirow{2}{*}{$p$ value } \\
\hline & $F$ & $p$ value & & & \\
\hline Tongue coating percentage (\%) & 3.195 & 0.079 & $34.79 \pm 10.76$ & $44.13 \pm 14.08$ & $0.004^{* *}$ \\
\hline \multicolumn{6}{|l|}{ Tongue body area } \\
\hline$L^{*}$ & 1.352 & 0.249 & $51.72 \pm 2.41$ & $50.50 \pm 2.83$ & 0.064 \\
\hline$a^{*}$ & 0.892 & 0.349 & $22.38 \pm 1.17$ & $22.26 \pm 1.61$ & 0.729 \\
\hline$b^{*}$ & 0.209 & 0.649 & $10.77 \pm 1.81$ & $11.39 \pm 2.02$ & 0.194 \\
\hline \multicolumn{6}{|l|}{ Whole tongue area } \\
\hline$L^{*}$ & 0.893 & 0.348 & $50.07 \pm 2.23$ & $49.51 \pm 2.79$ & 0.373 \\
\hline$a^{*}$ & 1.937 & 0.169 & $19.39 \pm 1.52$ & $18.21 \pm 2.06$ & $0.010^{* *}$ \\
\hline$b^{*}$ & 0.107 & 0.745 & $10.30 \pm 1.73$ & $10.84 \pm 2.00$ & 0.244 \\
\hline
\end{tabular}

The variables were compared by an independent samples $t$-test; ${ }^{* *} p<0.01$.

values between the two groups $(p=0.729)$. The differences in the tongue indices are presented in Table 2 and Figure 3.

\subsection{The Reliability of the Computerized Tongue Diagnostic Sys-} tem. Independent examination of two images per participant revealed the acceptable reliability of the CTDS, as shown in Table 3. All the ICCs of the tongue coating percentage and the $L^{*} a^{*} b^{*}$ values of the tongue body and whole body exceeded 0.8 (tongue coating percentage $=0.826$; the $\mathrm{TB} a^{*}$ value $=$ 0.801 ; the WT $a^{*}$ value $=0.812$ )

3.4. Binary Logistic Regression. We conducted binary logistic regression for $\mathrm{YD}$ with the tongue coating percentage and the WT $a^{*}$ value. Table 4 and Figure 4 show the regression model for the tongue coating percentage. The Wald test indicated that the tongue coating percentage was a significant predictor of YD $(p=0.007)$. In the regression model, the WT $a^{*}$ value was removed because it was not significant as a predictor of YD ( $p=0.759)$ once the tongue coating percentage was included in the model. The effective coating percentage for which there will be a $50 \%$ chance of YD is $39.47 \%(2.368 / 0.06)$.

3.5. Adverse Event. None of the patients experienced any adverse events during the study period.

\section{Discussion}

The results of the present study showed that the tongue coating percentage of the YD group was lower and that 


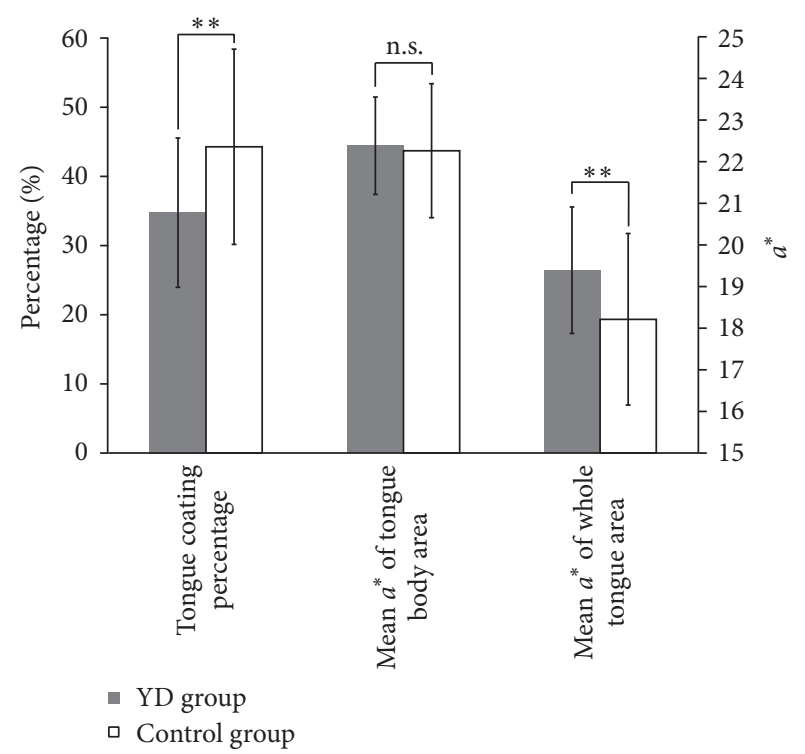

FIgURE 3: Comparison of the tongue indices between the Yindeficient group and the nondeficient control group. The tongue coating percentage of Yin-deficiency (YD) group (34.79\%) was significantly lower than that of the control group (44.13\%). The mean $a^{*}$ value of whole tongue area (WT $a^{*}$ ) was significantly different between the two groups; the WT $a^{*}$ value of the Yin-deficient group $(19.39 \pm 1.52)$ was higher than that of the control group $(18.21 \pm 2.06)$. However, the mean $a^{*}$ value of tongue body was not significantly different between the two groups. Vertical bars: $\pm \mathrm{SD} ;{ }^{* *} p<0.01$; n.s.: not significant.

TABLE 3: The reliability of the computerized tongue diagnostic system.

\begin{tabular}{lcc}
\hline & ICCs & Standard error $(95 \%$ CI $)$ \\
\hline $\begin{array}{l}\text { Tongue coating percentage } \\
\text { Tongue body area }\end{array}$ & 0.826 & $0.715-0.893$ \\
$\quad L^{*}$ & 0.800 & $0.673-0.877$ \\
$a^{*}$ & 0.801 & $0.675-0.878$ \\
$b^{*}$ & 0.865 & $0.779-0.917$ \\
Whole tongue area & & \\
$L^{*}$ & 0.805 & $0.682-0.881$ \\
$a^{*}$ & 0.812 & $0.694-0.885$ \\
$b^{*}$ & 0.876 & $0.797-0.924$ \\
\hline
\end{tabular}

the WT $a^{*}$ value was higher than those of the nondeficient control group. These results are consistent with the TKM tongue diagnosis theory that the tongue in patients with YD is reddened with little coating compared to the tongues of nondeficient individuals. However, we did not observe any significant difference in the TB $a^{*}$ values between the YD and control groups. This negative result suggests that a reddish color of the tongue is the result of a decrease in the tongue coating on the body of the tongue. Representative tongue image examples from the YD patient and the nondeficient control are shown in Figure 5.

Previous studies have found negative results when attempting to verify TKM and TCM theories. However, these

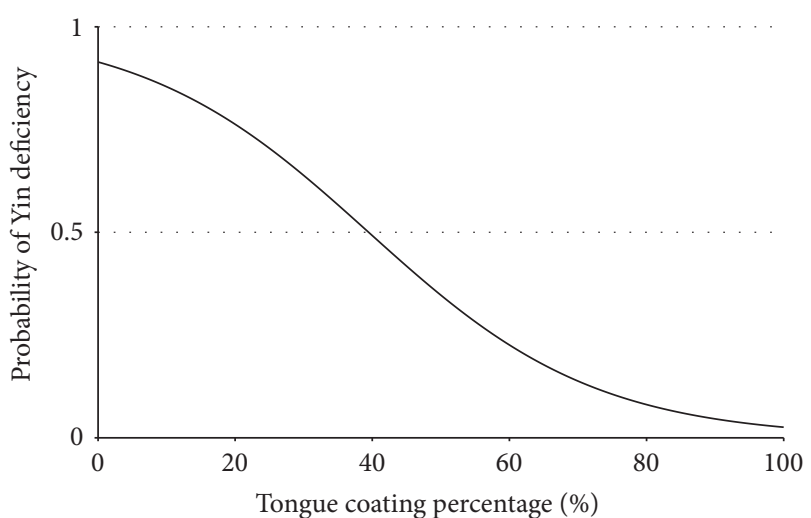

Figure 4: Logistic regression curve. Decrease of tongue coating percentage indicates an increased probability of Yin deficiency. [Probability of Yin deficiency $=\exp (0.06 X-2.368) /(1+\exp (0.06 X$ $-2.368)$ ); $X=$ tongue coating percentage (\%)].

negative results may have been due to the lack of objectivity in the methodology used in these studies; for most of these studies, the researchers subjectively evaluated the pattern and the tongue status, which likely biased their conclusions. In contrast, the present investigation used a more objective approach. We used two self-reporting questionnaires, in addition to the expert opinion of a practitioner to minimize the influence of experimenter bias, or the influence of an inaccurate self-report due to misunderstanding of the questionnaires. The patients that we identified as Yin-deficient complained of symptoms related to a YD; thus, our approach was likely objective and accurate.

Another strength of our study was the use of a CTDS to evaluate the tongue. With this approach, the conditions for obtaining tongue images remained constant. Moreover, our CTDS allowed a quantitative evaluation of tongue indices. The high ICC level demonstrates the reliability of our study.

In order to compare the tongue status with different patterns, the participants should have the same chief complaint but should be differentiated by variable patterns including YD. Therefore, we recruited patients who were all suffering from headache because the clinical pattern of a headache in TKM theory varies: wind-cold, wind-heat, wind-dampness, ascendant hyperactivity of the liver yang, static blood, heat syncope, dampness-phlegm, qi-deficiency, blood deficiency, and YD [26].

Previous studies have suggested that the amount of tongue coating increases with aging [27-30]; therefore, the participants in our study were limited to an age range of 20 to 49 years, and the control group was age-matched to the YD group. Consequently, the mean and standard deviation of age for both the YD group and the control group were similar. In addition, some studies have suggested that circadian rhythms influence oral status [31-33]; therefore, all of the examinations in our study were performed at approximately the same time in the morning. Moreover, our pilot study (not published) found that the correlation between the responses to the YD questionnaires and the tongue indices was weaker as time 


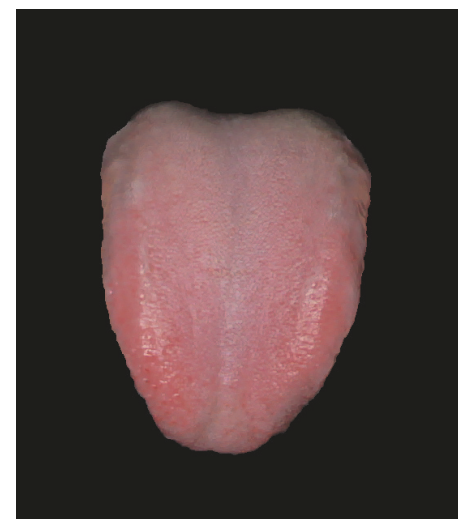

Score of YDQ: 598

Score of dichotomous YDS: 20

Tongue coating percentage: $9.72 \%$

Mean $a^{*}$ of tongue body area: 25.2

Mean $a^{*}$ of whole tongue area: 24.2

(a)

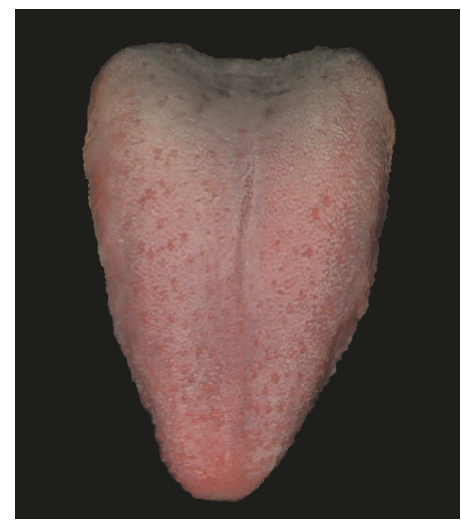

Score of YDQ: 35

Score of dichotomous YDS: 0

Tongue coating percentage: $43.82 \%$

Mean $a^{*}$ of tongue body area: 21.5

Mean $a^{*}$ of whole tongue area: 17.4

(b)

FIGURE 5: Representative tongue images from the Yin-deficient and control groups. A tongue coating percentage was used as an index to estimate the amount of tongue coating. The redness of the tongue was measured as the mean $a^{*}$ value of both the whole tongue area and the tongue body area that remained after excluding the tongue coating area. (a) A tongue image of a Yin-deficient patient is shown, and (b) a tongue image from a nondeficient control patient is shown. Note that these examples show that the tongue coating percentage of the Yin-deficient patient is lower and that the mean $a^{*}$ value of the whole tongue area is higher than those of the nondeficient patient. The mean $a^{*}$ value of the tongue body area showed a relatively small difference between the two samples.

TABLE 4: Binary logistic regression coefficients.

\begin{tabular}{lccccccc}
\hline Model & $B$ & SE & Wald & df & $p$ value & exp $(B)$ & $\begin{array}{c}\text { 95\% CI for exp }(B) \\
\text { Lower }\end{array}$ \\
\hline $\begin{array}{l}\text { Tongue coating } \\
\text { percentage }\end{array}$ & -0.060 & 0.022 & 7.354 & 1 & 0.007 & 0.941 & 0.901 \\
\begin{tabular}{l} 
Constant \\
\hline
\end{tabular} & 2.368 & 0.903 & 6.885 & 1 & 0.009 & 10.678 & 0.983 \\
\hline
\end{tabular}

progressed after completing the questionnaires; therefore, we conducted our examination within $24 \mathrm{~h}$ of the screening.

We also performed Pearson's correlation coefficient analysis to investigate relationships among the tongue indices and the YDQ and YDS scores. We found that the tongue coating percentage and test scores for YD were negatively correlated. Moreover, the WT $a^{*}$ values were positively correlated with the test scores for YD. However, this correlation was weak, as the correlation coefficients were between 0.25 and 0.35 . This result might be due to the lack of severity in the group of YD patients; the mean total YDQ scores and the dichotomized YDS score for the YD patients were not high (YDQ $=485.5$ \pm 94.4 ; YDS $=14.4 \pm 2.9$ ), considering that the highest scores achievable on these tests are 1,000 and 27, respectively. The WT $a^{*}$ values were very strongly correlated with the tongue coating percentage $(r=-0.922, p<0.001)$ and were not a significant predictor of YD when the tongue coating percentage was included in the logistic regression model. This implies that a "reddened tongue" can be interpreted as being the result of reduced coating on the tongue body, rather than the result of a reddened tongue body in the present study. Future studies should investigate patients with more severe YD to investigate whether a stronger correlation might appear.

The present study has several limitations. First, as mentioned above, the self-reporting screening could have been inaccurate due to the participant's misunderstanding of the questionnaire. Second, the number of participants that were included in our study was based on previous studies of tongue coating. Thus, our study design was more suitable for the investigation of tongue coating than of tongue color. Finally, although patterns of deficiency might be more easily observed in the elderly, the participants in the present study were relatively young, to increase the generalizability of the results.

\section{Conclusions}

Our results show that the tongue coating in self-reported YD patients tended to be decreased and the overall tongue color tended to be reddish compared to that in a nondeficient 
control group. These observations are consistent with traditional medicine theory of tongue diagnosis.
Abbreviations
CTDS: Computerized tongue diagnostic system
TB: $\quad$ Tongue body area
TB $a^{*}$ : The mean $a^{*}$ value for the tongue body area
TCM: Traditional Chinese medicine
TKM: Traditional Korean medicine
WT: Whole tongue area
WT $a^{*}$ : The mean $a^{*}$ value for the whole tongue area
YD: $\quad$ Yin deficiency
YDS: Yin-Deficiency Scale
YDQ: Yin-Deficiency Questionnaire.

\section{Conflicts of Interest}

The authors declare that there are no conflicts of interest regarding the publication of this manuscript.

\section{Acknowledgments}

This study was supported by the New Researcher Support Program through the National Research Foundation of Korea funded by the Ministry of Science, ICT \& Future Planning, Republic of Korea (NRF-2013R1A1A1059950), and by a grant of the Korea Health Technology R\&D Project through the Korea Health Industry Development Institute (KHIDI), funded by the Ministry of Health \& Welfare, Republic of Korea (HI15C0737). This paper is based on a study for the master's thesis of Kim [34].

\section{References}

[1] S. Lee, J. Park, H. Lee, and K. Kim, "Development and validation of Yin-Deficiency questionnaire," American Journal of Chinese Medicine, vol. 35, no. 1, pp. 11-20, 2007.

[2] B. Lee, Y. Park, and T. Kim, Diagnosis in Traditional Korean Medicine, Seongbosa, Seoul, Republic of Korea, 2000.

[3] WHO Regional Office for the Western Pacific, Standard Terminologies on Traditional Medicine in the Western Pacific Region, Renouf Publishing Co Ltd., Manila, Philippines, 2007.

[4] Z. L. Chen and Q. F. Hu, "Recent development in research on tongue inspection," Chinese Medical Journal, vol. 99, no. 6, pp. 444-456, 1986.

[5] S. Q. Zhang, "Tongue temperature of healthy persons and patients with Yin deficiency by using thermal video," Chinese Journal of Modern Developments in Traditional Medicine, vol. 10, no. 12, pp. 732-733, 1990.

[6] Q. F. Hu and Z. L. Chen, "Study on the mechanism of denuded tongue coating due to yin deficiency," Chinese Journal of Modern Developments in Traditional Medicine, vol. 9, no. 3, pp. 153-155, 1989.

[7] W. Su, Z. Y. Xu, Z. Q. Wang, and J. T. Xu, "Objectified study on tongue images of patients with lung cancer of different syndromes," Chinese Journal of Integrative Medicine, vol. 17, no. 4, pp. 272-276, 2011.

[8] S.-W. Park and D.-H. Nam, "A survey on utilization of questionnaires for assessing statues of Yin deficiency syndrome," The
Journal of the Society of Korean Medicine Diagnostics, vol. 18, no. 2, pp. 63-74, 2014.

[9] S.-C. Lin, M.-F. Chen, T.-C. Li, Y.-H. Hsieh, and S.-J. Liu, “The distribution of yin-deficient symptoms and their relationship on survival rate in cancer patients with Yin-Deficiency," American Journal of Chinese Medicine, vol. 36, no. 4, pp. 655-663, 2008.

[10] S.-C. Lin, M.-L. Huang, S.-J. Liu, Y.-F. Huang, S.-C. Chiang, and M.-F. Chen, "Severity of Yin deficiency syndrome and autonomic nervous system function in cancer patients," Journal of Alternative and Complementary Medicine, vol. 15, no. 1, pp. 87-91, 2009.

[11] S.-C. Lin and M.-F. Chen, "Increased yin-deficient symptoms and aggravated autonomic nervous system function in patients with metastatic cancer," Journal of Alternative and Complementary Medicine, vol. 16, no. 10, pp. 1059-1063, 2010.

[12] M. Kim, D. Cobbin, and C. Zaslawski, “Traditional Chinese medicine tongue inspection: an examination of the inter- and intrapractitioner reliability for specific tongue characteristics," Journal of Alternative and Complementary Medicine, vol. 14, no. 5, pp. 527-536, 2008.

[13] M. M. Ko, J. A. Lee, B.-K. Kang, T.-Y. Park, and M. S. Lee, "Interobserver reliability of tongue diagnosis using traditional Korean medicine for stroke patients," Evidence-Based Complementary and Alternative Medicine, vol. 2012, Article ID 209345, 6 pages, 2012.

[14] J. Kim, G. Han, S.-J. Ko et al., “Tongue diagnosis system for quantitative assessment of tongue coating in patients with functional dyspepsia: a clinical trial," Journal of Ethnopharmacology, vol. 155, no. 1, pp. 709-713, 2014.

[15] I. Gareus, T. Rampp, L. Tan et al., "Introducing a computerassisted, digital tongue-imaging device for standardization of traditional tongue diagnosis," Forschende Komplementarmedizin, vol. 21, no. 3, pp. 190-196, 2014.

[16] G. Han, J.-W. Park, S.-J. Ko et al., "Study on tongue coating patterns of the xerostomia in the elderly patients," The Journal of the Society of Korean Medicine Diagnostics, vol. 17, no. 3, pp. 189-202, 2013.

[17] Y.-J. Park, S.-W. Cho, B.-H. Lee, and Y.-B. Park, "Development and validation of the yin deficiency scale," Journal of Alternative and Complementary Medicine, vol. 19, no. 1, pp. 50-56, 2013.

[18] S. Lee, J. Park, and K. Kim, "Development of Yin-deficiency questionnaire and examine the reliability and validity," Korean Journal of Oriental Physiology \& Pathology, vol. 18, no. 2, pp. 376-380, 2004.

[19] J. Kim, J. Kim, J. Park, and B. Ryu, "Availability of diagnosis of yin-deficiency in elderly people with xerostomia and factors influencing subjective oral dryness: a prospective crosssectional study," Journal of Korean Medicine, vol. 34, no. 3, pp. 13-24, 2013.

[20] J. Kim, J. Jo, S. Yoo et al., "A study on relations between MRS, MENQOL, HRV, Yin-deficiency questionnaire in menopausal woman with hot flush," The Journal of Oriental Obstetrics \& Gynecology, vol. 24, no. 4, pp. 71-84, 2011.

[21] G. Cho, S. Yu, E. Kim et al., "Change of Yin-deficiency scale and the skins physical properties based on female's aging pattern by 7 years," The Journal of Korean Medical Institute of Dermatology \& Aesthetics, vol. 3, no. 1, pp. 24-35, 2010.

[22] S. Jang and J. Kim, "Development cut-off value for Yin-deficiency questionnaire and diagnositic ability of Yin-deficiency in xerostomia," Journal of Internal Korean Medicine, vol. 35, no. 4, pp. 483-497, 2014. 
[23] J. Kim, J. Son, S. Jang et al., "Availability of tongue diagnosis system for assessing tongue coating thickness in patients with functional dyspepsia," Evidence-Based Complementary and Alternative Medicine, vol. 2013, Article ID 348272, 6 pages, 2013.

[24] A. Agresti, Categorical Data Analysis, Wiley Series in Probability and Mathematical Statistics, John Wiley \& Sons, New York, NY, USA, 2013.

[25] P. E. Shrout and J. L. Fleiss, "Intraclass correlations: uses in assessing rater reliability," Psychological Bulletin, vol. 86, no. 2, pp. 420-428, 1979.

[26] The Association of Department of Korean Heart Internal Medicine, Cardiology and Neurology of Korean Traditional Internal Medicine, Koonja Publishing Inc, Seoul, Republic of Korea, 4th edition, 2011.

[27] W. J. Ralph, "Hygiene of the tongue," Gerodontics, vol. 3, no. 4, pp. 169-170, 1987.

[28] W. J. Ralph, “Oral hygiene-why neglect the tongue?” Australian Dental Journal, vol. 33, no. 3, pp. 224-225, 1988.

[29] K. Yaegaki and K. Sanada, "Volatile sulfur compounds in mouth air from clinically healthy subjects and patients with periodontal disease," Journal of Periodontal Research, vol. 27, no. 4, pp. 233-238, 1992.

[30] M. Quirynen, C. Mongardini, and D. Van Steenberghe, "The effect of a 1-stage full-mouth disinfection on oral malodor and microbial colonization of the tongue in periodontitis patients. A Pilot Study," Journal of Periodontology, vol. 69, no. 3, pp. 374382, 1998.

[31] H. Miyazaki, S. Sakao, Y. Katoh, and T. Takehara, "Correlation between volatile sulphur compounds and certain oral health measurements in the general population," Journal of Periodontology, vol. 66, no. 8, pp. 679-684, 1995.

[32] K. Ikawa, M. Iwakura, J. Washio, A. Kusano, N. Tanda, and T. Koseki, "Circadian changes of volatile sulfur compounds measured by Breathtron ${ }^{\mathrm{TM}}$," International Congress Series, vol. 1284, pp. 89-90, 2005.

[33] S. Supriatno, "The effect of circadian rhythm to Oral volatile sulfur compounds production," Majalah Kedokteran Gigi Indonesia, vol. 20, no. 1, pp. 14-20, 2013.

[34] S. Kim, Comparative analysis of tongue indices between patients with and without a self-reported Yin-deficiency: a cross-sectional study [M.S. thesis], Sangji University, 2017. 


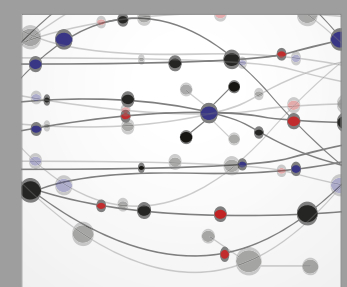

The Scientific World Journal
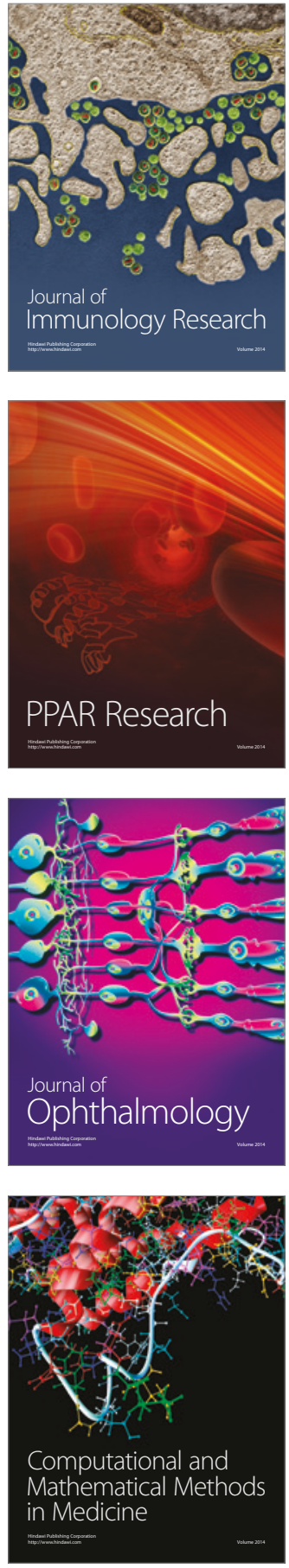

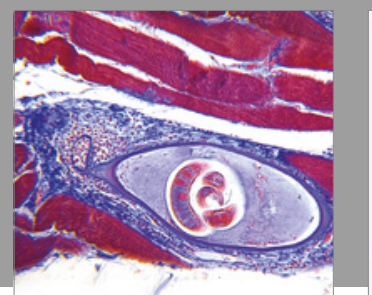

Gastroenterology Research and Practice
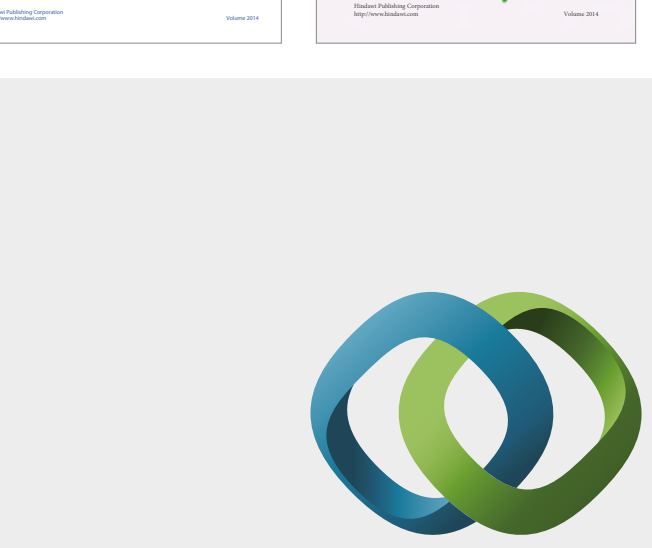

\section{Hindawi}

Submit your manuscripts at

https://www.hindawi.com
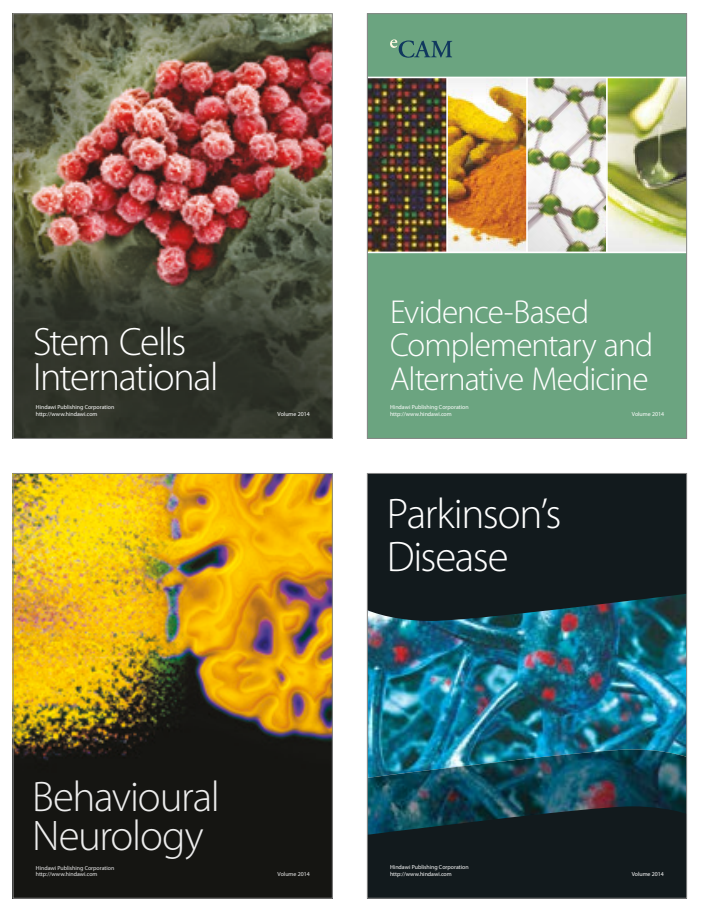
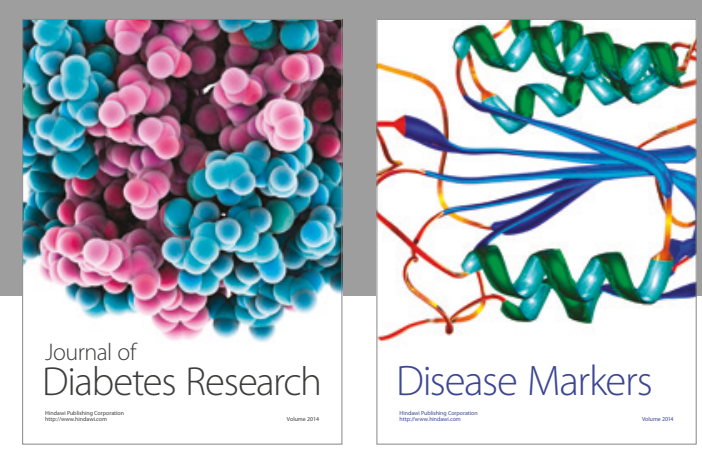

Disease Markers
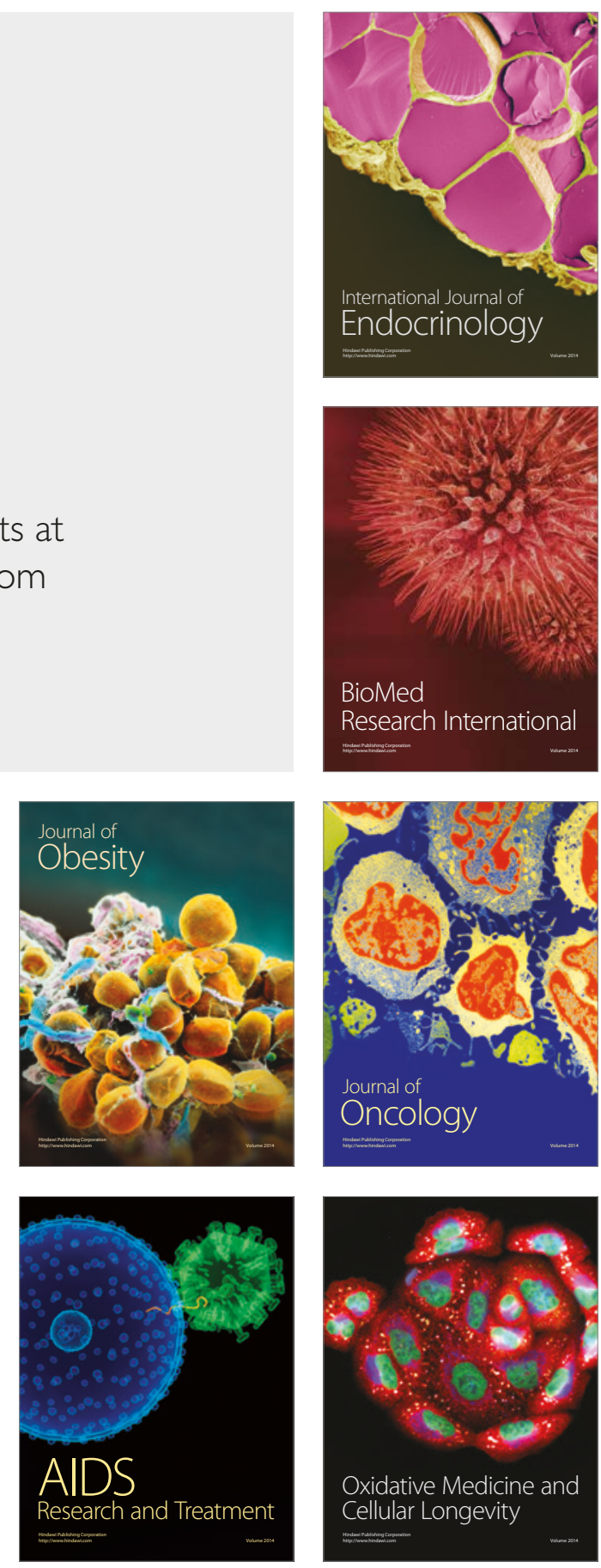